\title{
Pressure profile similarities between tongue resistance training tasks and liquid swallows
}

\author{
Catriona M. Steele, PhD; ${ }^{1-5 *}$ Gemma L. Bailey, MHSc; ${ }^{1}$ Sonja M. Molfenter, MHSc; ${ }^{1}$ Erin M. Yeates, MHSc; $^{1}$ \\ Karen Grace-Martin, $\mathbf{M A}^{6}$ \\ ${ }^{1}$ Toronto Rehabilitation Institute, Toronto, Canada; ${ }^{2}$ Department of Speech-Language Pathology, ${ }^{3}$ Institute of Bioma- \\ terials and Biomedical Engineering, and ${ }^{4}$ Graduate Department of Rehabilitation Sciences, University of Toronto, \\ Toronto, Canada; ${ }^{5}$ Bloorview Kids Rehab, Toronto, Canada; ${ }^{6}$ The Analysis Factor, Ithaca, NY
}

\begin{abstract}
Tongue-pressure resistance training is known to increase tongue strength in seniors and individuals with strokerelated dysphagia. However, evidence of associated functional improvements in swallowing is equivocal. We investigated similarities in pressure waveform profiles between swallowing and several tongue-palate pressure tasks to identify tasks that may be best suited for inclusion in tongue-pressure resistance training protocols for patients who are unable to safely perform real bolus swallows in treatment. Tongue-palate pressures were recorded in 20 healthy young adults. Participants performed water and nectarthick juice swallows, effortful and noneffortful saliva swallows, and "half-maximum" tongue-palate partial-pressure tasks emphasizing either anterior or posterior tongue-palate contact at different speeds. Pressure slopes (amplitude change over time) during the pressure application (rise) and withdrawal (release) phases were analyzed. A subset of four tasks with the greatest similarity in slope characteristics to those seen in bolus swallows was identified: anterior-emphasis half-maximum tongue-palate presses, posterior-emphasis maximum isometric tongue-palate presses, posterior-emphasis half-maximum slow tongue-palate presses, and effortful saliva swallows. We propose that future research should explore the degree to which swallowing improvements are obtained from treatment protocols that emphasize these tasks.
\end{abstract}

Key words: dysphagia, exercise, oral-motor, pressure, rehabilitation, resistance, speech-language pathology, swallowing, tongue, tongue resistance training.

\section{INTRODUCTION}

Tongue-pressure resistance training has recently emerged as a treatment approach with the potential to yield positive outcomes in adults with swallowing impairment involving tongue weakness. Both healthy seniors and individuals with stroke-related dysphagia were found to experience significant gains in measures of maximum isometric tongue strength following an 8-week course of resistance exercise in which treatment focused on achieving strength targets in the range of 60 to 80 percent of maximum isometric pressure capacity [1-2].

\footnotetext{
Abbreviations: AHMAX = anterior-emphasis half-maximum tongue-palate press, AHMAXFAST $=$ AHMAX performed at fast rate, AHMAXSLOW = AHMAX performed at slow rate, ANOVA $=$ analysis of variance, $d f=$ degrees of freedom, DSANEC = discrete nectar-thick apple juice swallow, DSW = discrete water swallow, ESS = effortful saliva swallow, IOPI = Iowa Oral Performance Instrument, NESS = noneffortful saliva swallow, PHMAX = posterior-emphasis half-maximum tonguepalate press, PHMAXFAST $=$ PHMAX performed at fast rate, PHMAXSLOW $=$ PHMAX performed at slow rate, PMAXTP $=$ posterior-emphasis maximum isometric tongue-palate press.

* Address all correspondence to Catriona M. Steele, PhD; Toronto Rehabilitation Institute, 550 University Avenue, 12030, Toronto, ON, M5G 2A2, Canada; 416-597-3422, ext 7603. Email: steele.catriona@torontorehab.on.ca

DOI:10.1682/JRRD.2009.05.0068
} 
However, evidence that these improvements generalize to swallowing remains equivocal. In a small sample of 10 stroke patients, Robbins et al. reported faster oral transit times and improved penetration-aspiration scores on thin liquid swallowing tasks at posttreatment videofluoroscopy [2], but changes in other impairments, such as pharyngeal residue, remained elusive.

Within the speech-language pathology literature, interest in exercise-based approaches to speech and swallowing rehabilitation has been growing [1-8]. Proponents of an exercise-based approach advise that the basic tenants of exercise-based physical rehabilitation must be applied to speech and swallowing goals to afford the best chance of successful outcomes [4,8]. First, treatment should employ tasks that are highly specific to the task for which functional outcomes are desired (i.e., swallowing). Second, exercises must be practiced with sufficient intensity to induce fatigue. Finally, exercise must be practiced with sufficient frequency for sufficient duration to be likely to induce muscle changes. The previous studies by Robbins et al. adhere to the latter two of these principles: they target tongue strength changes by including 60 task repetitions daily, in the 60 to 80 percent range of maximum capacity, on nonconsecutive days over an 8-week period [1-2]. Furthermore, the Robbins et al. protocol addresses the possibility that resistance exercises should selectively target different regions of the tongue by dividing the exercise tasks equally into anterior-emphasis and posterior-emphasis tongue-palate press tasks. For the anterior-emphasis tasks, patients are instructed to place a pressure bulb in the front of the mouth and squeeze the bulb by using the front of the tongue. For the posterioremphasis tasks, the pressure bulb is positioned farther back in the mouth and patients are instructed to squeeze the bulb by using the back of the tongue. However, the Robbins et al. exercise protocol focuses exclusively on strength and does not include actual swallowing tasks or other possible tongue-press tasks, such as those emphasizing precision, endurance, or speed. The fact that swallowing pressures are known to fall well short of those pressures registered in maximum isometric tasks [9] calls into question the emphasis on strength goals and outcomes in this approach.

In our laboratory, we have been studying patterns of tongue-pressure application in swallowing and exploring tongue-pressure resistance training as a method for effective swallow rehabilitation. Our primary goal is to restore functional swallowing with thin liquids. We have been particularly interested in the possible contribution of motor skill to tongue-pressure application, inspired by evidence from other rehabilitation literature that skill training (involving the repeated practice of goal-oriented tasks) can lead to faster and more sustainable changes in motor function than strength training alone [10]. Skilled movements are defined as those movement tasks that require the modification and organization of muscle synergies into effective movement sequences [11]. Our interest in this concept led us to develop a tongue resistance training protocol that involves repeated practice of submaximal partial-pressure generation tasks, with an emphasis on pressure precision [12]. The lack of clear evidence of generalization from improved pressure outcomes to improved swallowing outcomes in these cases prompted us to investigate pressure profiles more closely and explore the role of timing [13-14] (and temporal modulation) as a factor in tongue-pressure behavior in swallowing. Systematic variations in the temporal aspects of tongue-pressure onset and withdrawal between water and nectar-thick juice swallows have recently been described in healthy young adults [13]. The rise and release phases of tongue-palate pressure behavior may logically be considered to have primary salience for propulsion of a bolus through the mouth toward the pharynx in swallowing [14-16]. This observation supports the speculation that treatment tasks that most closely match the slope characteristics (i.e., amplitude changes over time) of both tongue-pressure application and release in healthy swallowing may be optimal tasks to include in a program of swallowing rehabilitation. Although true task specificity for swallowing would require actual swallows, the idea that some nonswallowing tongue-press tasks might closely mirror critical aspects of the tonguepressure motor pattern seen in swallowing is important given that many patients who aspirate may be unable to safely include bolus swallows in their swallowing rehabilitation regime.

The goal of the current study was to explore similarities in tongue-pressure profile between liquid swallowing tasks and various tongue-press tasks in healthy adults on the basis of three specific criteria: (1) whether pressures are registered in the same locations on the palate, (2) the rate of amplitude change at the anterior palate during the pressure onset phase (rise slope), and (3) the rate of amplitude change at the posterior palate during the pressure release phase (release slope) (Figure 1). For the current study, we studied partial-pressure amplitude targets in the 


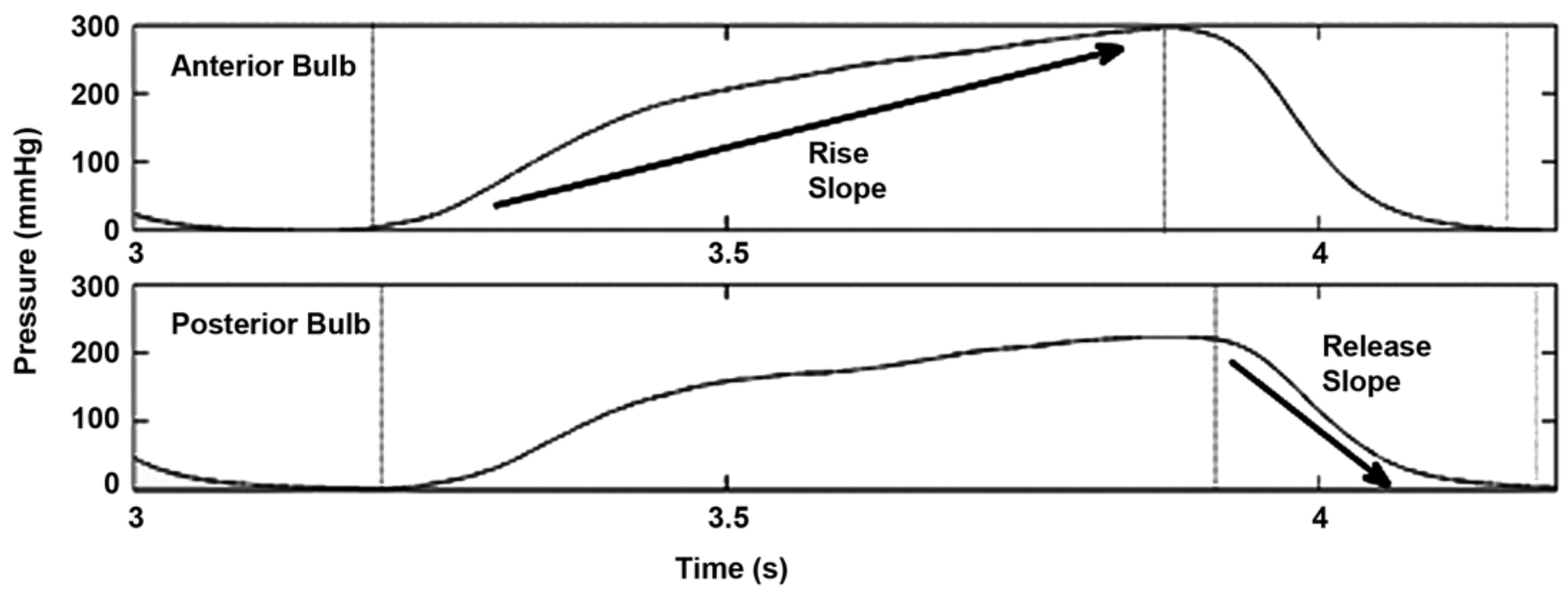

Figure 1.

Anterior and posterior pressure-bulb waveforms during anterior tongue-palate press task. Onsets, offsets, and peak pressures are denoted in each waveform by dashed vertical lines. Portion of anterior bulb waveform from pressure onset until peak pressure is the rise phase. Portion of posterior bulb waveform following peak until return to signal baseline is the release phase. Pressure slope for these phases was calculated as change in signal amplitude (in millimeters of mercury) divided by phase duration (in seconds).

range of 50 percent of each participant's maximum isometric pressure capacity (henceforth called "half-maximum tongue-palate press" tasks). The choice of this target range was motivated by previous evidence showing that habitual swallowing pressures typically fall in or below this halfmaximum range [9].

Discrete liquid swallowing tasks were performed with two stimuli: water (discrete water swallow [DSW]) and nectar-thick apple juice (discrete nectar-thick apple juice swallow [DSANEC]). Comparator tasks included anterioremphasis tasks, for which the front of the tongue is pressed up against the front of the palate (the alveolar ridge, just behind the upper incisors), and posterior-emphasis tasks, for which the back of the oral tongue is pressed up against the back of the palate (targeting the anatomical region around the transition from the hard to soft palate). Task instructions for the following comparator tasks are detailed in Table 1: anterior-emphasis half-maximum tongue-palate press (AHMAX), posterior-emphasis maximum isometric tonguepalate press (PMAXTP), posterior-emphasis half-maximum tongue-palate press (PHMAX), noneffortful saliva swallow (NESS) [13,15], and effortful saliva swallow (ESS) [13,15]. The half-maximum tongue-palate press tasks were conducted at three rates: habitual (AHMAX, PHMAX), fast (AHMAX performed at fast rate [AHMAXFAST], PHMAX performed at fast rate [PHMAXFAST]), and slow (AHMAX performed at slow rate [AHMAX-SLOW], PHMAX performed at slow rate [PHMAXSLOW]).

\section{METHODS}

\section{Participants}

Twenty healthy adults (10 male, 10 female) under the age of 40 provided written consent for participation in the study. Participants attended an intake session in which an oral-mechanism examination and a swallow screening were performed to rule out clinical signs of swallowing difficulty. Participants with known histories of swallowing difficulties, stroke, brain injury, neurodegenerative disease, or head and neck cancer were excluded.

Data were collected with use of the orolingual manometry module of the KayPentax Digital Swallow Workstation (KayPentax; Lincoln Park, New Jersey). A soft plastic strip containing three silicon air bulbs (diameter: $13 \mathrm{~mm}$; interbulb spacing: $8 \mathrm{~mm}$ ) was adhered to each participant's palate in midline with a small amount of a cyanoacrylate dental adhesive (Iso-Dent, Ellman International Inc; Oceanside, New York). The strip was positioned such that the anterior bulb was located immediately behind the front 
JRRD, Volume 47, Number 7, 2010

Table 1.

Experimental instructions for swallow and tongue-press tasks.

\begin{tabular}{|c|c|}
\hline Task & Instruction \\
\hline $\begin{array}{l}\text { Anterior-Emphasis Maximum } \\
\text { Isometric Tongue-Palate Press }\end{array}$ & $\begin{array}{l}\text { Press the front of your tongue up to the front of your palate (touching the bony ridge just } \\
\text { behind your upper teeth) as hard as you can. We will be calling this the "front position." }\end{array}$ \\
\hline $\begin{array}{l}\text { Discrete Nectar-Thick Apple } \\
\text { Juice Swallow }\end{array}$ & Take and swallow one sip of this thick apple-juice, then remove the cup from your lips. \\
\hline Effortful Saliva Swallow & Do a "hard" saliva swallow. Imagine that you are swallowing a whole grape. \\
\hline $\begin{array}{l}\text { Anterior-Emphasis Half-Maximum } \\
\text { Tongue-Palate Press (AHMAX) }\end{array}$ & $\begin{array}{l}\text { In the front position you were able to hit a } \_\mathrm{kPa} \text { on your maximum effort tongue press. } \\
\text { Now I want you to aim for half of that value. So, try to hit a } \_ \text {kPa in the front position. }\end{array}$ \\
\hline AHMAX Performed at Slow Rate & $\begin{array}{l}\text { Your half-maximum target value in the front position is __ } \mathrm{kPa} \text {. We now want you to com- } \\
\text { plete this task slowly. We want you to gradually reach __ } \mathrm{kPa} \text {. Try to make the numbers } \\
\text { move up one at a time. }\end{array}$ \\
\hline $\begin{array}{l}\text { Posterior-Emphasis Maximum } \\
\text { Isometric Tongue-Palate Press }\end{array}$ & $\begin{array}{l}\text { Now you are going to perform a maximum effort press in the back position. Move the bulb } \\
\text { about an inch further back from the front position. Then push straight up with the body of } \\
\text { your tongue and squeeze the air out of the bulb against the roof of your mouth. Squeeze as } \\
\text { hard as you can. }\end{array}$ \\
\hline $\begin{array}{l}\text { Posterior-Emphasis Half-Maximum } \\
\text { Tongue-Palate Press (PHMAX) }\end{array}$ & $\begin{array}{l}\text { In the back position, you were able to hit a _ } \mathrm{kPa} \text { on your maximum effort tongue press. } \\
\text { Now I want you to aim for half of that value. So, try to hit a __ } \mathrm{kPa} \text { in the back position. }\end{array}$ \\
\hline PHMAX Performed at Slow Rate & $\begin{array}{l}\text { Your half-maximum target value in the back position is __ } \mathrm{kPa} \text {. We now want you to com- } \\
\text { plete this task slowly. We want you to gradually reach __ } \mathrm{kPa} \text {. Try to make the numbers } \\
\text { move up one at a time. }\end{array}$ \\
\hline
\end{tabular}

teeth. Pressures from the anterior, medial, and posterior palatal bulbs were registered on a monitor in a window with a $500 \mathrm{mmHg}$ maximum and sampled at $250 \mathrm{~Hz}$. For a few participants, maximum pressures obviously exceeded $500 \mathrm{mmHg}$, in which case the equipment was adjusted to allow a maximum of $750 \mathrm{mmHg}$. Equipment was calibrated at the beginning of each recording session as per manufacturer's instructions. Before the tongue-bulb array was attached, task training was provided. Participants were taught to perform maximum anterior- and posterior-emphasis tongue presses, and immediate feedback regarding pressure values was provided with use of the Iowa Oral Performance Instrument (IOPI). Participants were then instructed in the performance of half-maximum tongue-pal- ate press tasks, again with use of the IOPI for biofeedback. The experiment then proceeded with use of the KayPentax equipment to register tongue-palate pressures. Participants were instructed to perform the 10 different experimental tasks in randomized order, with five repetitions per task, to enable the calculation of representative-task mean data values for each participant. Previous literature regarding intraparticipant variability in swallowing suggests that at least three repetitions of a given swallowing task are required to obtain representative tongue-pressure measures [17].

Instructions were displayed on a computer monitor in front of the participant so that mandatory quiet rest periods could be interspersed between tasks. For the saliva swallow tasks, one swallow was cued every 30 seconds, 
for a total signal recording time of 2 minutes 30 seconds per task. This ensured a rest period between swallows to allow for saliva replenishment. The bolus-swallow and tongue-press tasks were performed at a natural pace, unless the task instructions specifically indicated that the participant was to perform rapid or slow half-maximum tongue-palate presses. For the bolus-swallow tasks, participants were provided with a cup containing approximately $150 \mathrm{~mL}$ of water or nectar-thick apple-juice (Resource, Nestlé Nutrition; Highland Park, Michigan) and cued to take five comfortably sized sips of liquid in a row from the cup, perform one discrete swallow per sip, and then remove the cup from their lips before the next swallow. Precise sip-volume measurement techniques cannot easily be employed during the collection of natural-reiterated discrete swallowing data. However, on the basis of previous studies using these same tasks, we can assume that sip size in this study fell between 5 and $10 \mathrm{~mL}$ per swallow and likely varied less than $2 \mathrm{~mL}$ from swallow to swallow within a participant [18].

\section{Data Processing}

The first step in data processing was to index important events in the pressure waveforms. This task was completed by two trained research associates, who aligned a cursor in the KayPentax manometry software with the onset, peaks, and offsets of pressure events in each pressure waveform. For each event, the time point (in milliseconds) and pressure amplitude (in millimeters of mercury) were recorded in a spreadsheet for subsequent analysis. Ten percent of the data were indexed in duplicate for calculation of interrater agreement, which was excellent (intraclass correlation $=1.00$ ). . Due to a technical problem with the pressure measurement equipment, data for one male participant could not be included in the analysis. Pressure amplitude differences in millimeters of mercury (henceforth called "range”), duration (in milliseconds), and slopes (range divided by duration, in millimeters of mercury per second) were calculated for the anterior pressure-bulb rise phase (pressure onset to pressure peak at the anterior bulb) and posterior pressure-bulb release phase (pressure peak to pressure offset at the posterior bulb) of each pressure event. Before further analysis, all range data were normalized relative to a standardized value of $600 \mathrm{mmHg}$ assigned to the maximum range of anterior-emphasis maximum isometric tongue-palate pressure rise registered at the anterior pressure bulb for each participant: normalized pressure range $=$ (pressure range/ maximum isometric pressure range) $\times 600$.
Normative values for maximum isometric pressures at the anterior palate have previously been reported to fall at approximately $600 \mathrm{mmHg}$ for healthy young adults [9].

\section{Analysis}

The analysis for this study was conducted in a stepwise fashion. Chi-square statistics were used to compare the frequency with which activation of both the anterior and posterior pressure bulbs occurred in the different tasks included in the study. Descriptive statistics for rise slope and release slope were examined and Forest plots showing 95 percent confidence interval overlap across tasks were prepared (Figures 2 and 3). To further examine task differences statistically, we ran two mixed-model analysis of variance (ANOVA) models (rise slope, release slope) using the mixed procedure in SPSS 16.0 (SPSS, Inc; Chicago, Illinois). Both models controlled

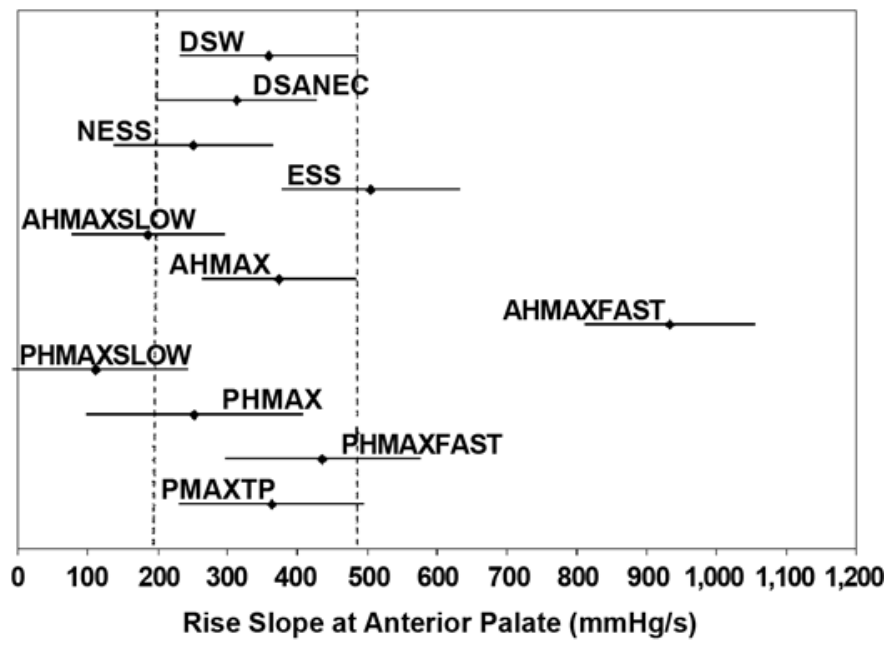

Figure 2.

Forest plot showing overlap in mean and 95\% confidence interval for tongue-pressure rise slope, measured at anterior palate, during 11 different tasks: discrete water swallow (DSW), discrete nectar-thick apple juice swallow (DSANEC), noneffortful saliva swallow (NESS), effortful saliva swallow (ESS), anterior-emphasis half-maximum tongue-palate press (AHMAX), AHMAX performed at slow rate (AHMAXSLOW), AHMAX performed at fast rate (AHMAXFAST), posterior-emphasis half-maximum tongue-palate press (PHMAX), PHMAX performed at slow rate (PHMAXSLOW), PHMAX performed at fast rate (PHMAXFAST), and posterior-emphasis maximum isometric tongue-palate press (PMAXTP). Dashed lines outline interval capturing rise-slope values for DSW and DSANEC. Tasks with mean and confidence interval boundaries falling outside these lines differ from these bolus swallowing tasks in rise slope. 


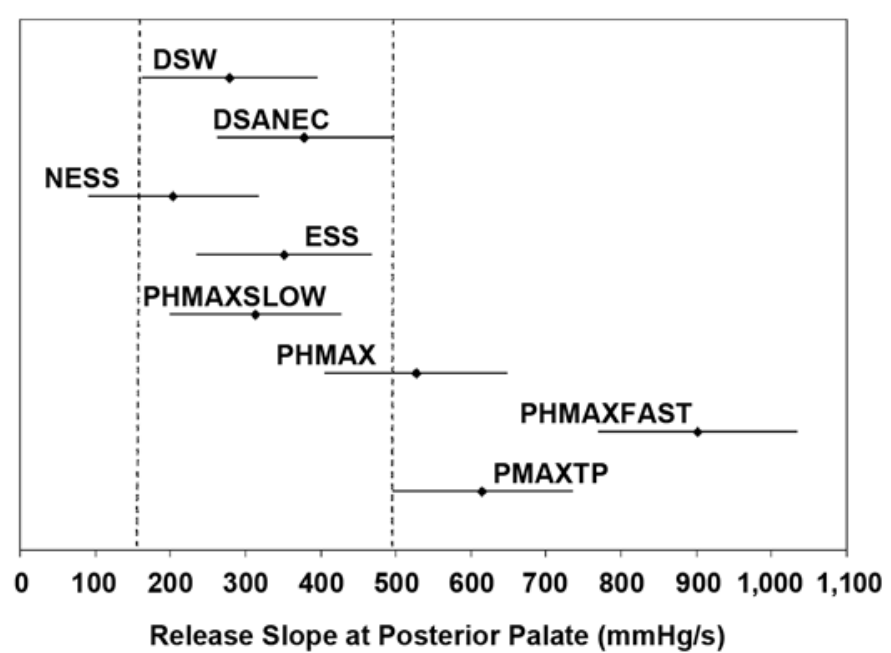

Figure 3.

Forest plot showing the overlap in mean and 95\% confidence interval for tongue-pressure release slope, measured at posterior palate, during eight different tasks: discrete water swallow (DSW), discrete nectar-thick apple juice swallow (DSANEC), noneffortful saliva swallow (NESS), effortful saliva swallow (ESS), posterior emphasis half-maximum tongue-palate press (PHMAX), PHMAX performed at slow rate (PHMAXSLOW); PHMAX performed at fast rate (PHMAXFAST), and posterior-emphasis maximum isometric tongue-palate press (PMAXTP). Dashed lines outline interval capturing release-slope values for DSW and DSANEC. Tasks with mean and confidence interval boundaries falling outside these lines differ from these bolus swallowing tasks in release slope.

for the within-subject nature of the 11 tasks by including random effects for participant and participant $\times$ task interaction, with a variance components covariance structure and restricted maximum likelihood estimation. Furthermore, rather than using the mean response of the five replicate trials for each swallowing task as the experimental unit, which ignores variability among replications, we captured the variation among the trials directly by modeling the variance-covariance matrix of the residuals on each task for each participant. The best-fitting covariance structure for the residuals across trials was a diagonal structure, which estimated a unique variance for each trial but had no additional correlation among these residuals. Autoregressive and compound symmetry covariance structures were tested, but neither resulted in improved fit of the model, so the simpler diagonal structure was retained (-2RLL [restricted log likelihood] = $7,607.58)$. Sex was included as a fixed, between-subjects factor to both models, but there were no significant main effects in either one (rise slope: $F(1,14.4)=0.11, p=$ 0.74 ; release slope $F(1,16.47)=0.354, p=0.56)$. Consequently, the sex factor was dropped from the model. Post hoc tests compared each of the nine pressure tasks to each of the two bolus swallowing tasks (DSW and DSANEC) by using Sidak adjustments to control for Type I error. The nine pressure tasks were not compared to one other, since these comparisons were not of interest.

\section{RESULTS}

The DSW and DSANEC tasks typically elicited activation of both the anterior and posterior pressure bulbs. The saliva swallow tasks presented with this same activation pattern at least 94 percent of the time. By contrast, the AHMAX tasks were significantly less likely to display activation of both bulbs $\left(\chi^{2}\right.$, degrees of freedom [ $\left.d f\right]$ $10,=424.43, p<0.001)$ and involved isolated activation of the anterior bulb at least 84 percent of the time. The PMAXTP task most commonly elicited coactivation of both bulbs (77\% of the time), with the remaining cases involving isolated posterior-bulb activity. The PHMAX tasks involved coactivation of both bulbs between 50 and 56 percent of the time and isolated posterior activation 35 to 47 percent of the time.

The activation-pattern comparison suggested that AHMAX tasks were dissimilar from swallowing tasks in their tongue-pressure profiles. This finding was further confirmed in the descriptive statistics (Table 2) and the Forest plot comparisons of rise- and release-slope confidence interval overlaps. These results suggested that the AHMAX and PMAXTP tasks had similar rise slopes to the DSW and DSANEC (Figure 2), while the ESS and PHMAXSLOW tasks overlapped with the DSW and DSANEC for release slope (Figure 3).

In the mixed-model ANOVAs, a significant main effect of task was found in the rise-slope data $(F(10$, $129.18)=12.74, p<0.001)$. The AHMAXFAST task had a significantly greater rise slope than both the DSW ( $p<$ $0.001)$ and DSANEC $(p<0.001)$. None of the other tasks showed significant differences in pairwise comparisons to the DSW and DSANEC.

A significant main effect of task was also found in the release-slope data $(F(7,82.52)=16.78, p<0.001)$. The smaller value for the error $d f$ in this analysis reflects exclusion of the AHMAX tasks from the model, based on the fact that these involved no activation of the posterior 
Table 2.

Tongue-pressure profiles for swallow and tongue-press tasks. Data shown as mean \pm standard deviation.

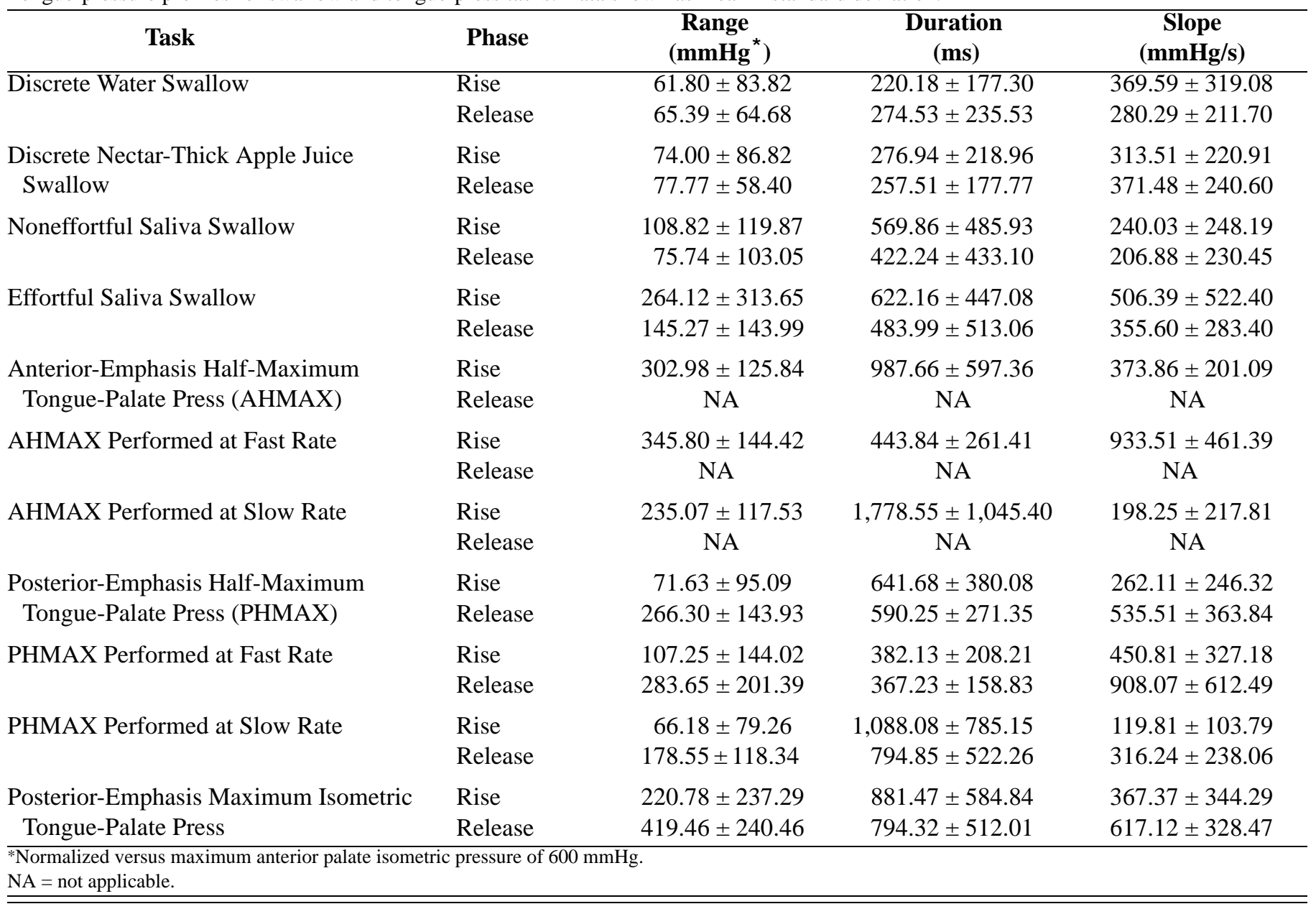

pressure bulb. Post hoc pairwise comparisons identified significant differences between the DSW and the PHMAX $(p<0.007)$, PHMAXFAST $(p<0.001)$, and PMAXTP $(p<0.001)$ tasks. Significant pairwise differences from the DSANEC were also found for the PHMAXFAST $(p<0.001)$ and PMAXTP $(p<0.01)$ tasks.

In summary, all tasks other than the AHMAXFAST task were found to have statistical similarity (or nondifference) from DSW and DSANEC with respect to rise slope. Of these, inspection of confidence interval overlaps suggests that the AHMAX and PMAXTP tasks have rise-slope values that fall closest to those seen in liquid swallowing tasks. With respect to release slope, clear differences were noted between liquid swallowing tasks and the AHMAX (habitual, slow, and fast rates), PHMAX, PHMAXFAST, and PMAXTP tasks. This leaves the NESS and ESS tasks and the PHMAXSLOW task, which demonstrated statistical nondifference from the liquid swallowing tasks. Of these, confidence interval overlaps were strongest for the ESS and PHMAXSLOW tasks.

\section{DISCUSSION}

The primary goal of this study was to identify those tongue resistance training tasks that most closely resemble bolus swallows in their pressure-slope rise and release characteristics so that these tasks might become a focus in future swallowing treatment studies. We have demonstrated that in healthy young adults, the rise-slope characteristics of AHMAX tasks and PMAXTP tasks are most similar to those seen in DSW and DSANEC. Similarly, the slope characteristics of pressure release in saliva swallowing tasks (noneffortful and effortful) and PHMAXSLOW 
tasks are similar to those seen in liquid swallowing tasks. As an outcome of this study, we propose that future research should explore the potential of treatment protocols emphasizing these tasks (i.e., with slope profiles that are not significantly different from those seen in healthy swallowing) to yield improvements that generalize to functional swallowing. One must, of course, recognize that the absence of significant differences in an ANOVA test cannot be interpreted to imply equivalence across the tasks compared in this study. The current findings, therefore, represent preliminary evidence that requires further substantiation.

Interestingly, the pressure ranges registered during DSW and DSANEC tasks in this experiment fell well short of maximum isometric pressure values (normalized to a value of $600 \mathrm{mmHg}$ ), consistent with previous studies [9]. This phenomenon appears to have occurred to a greater degree in our research participants than previously described in the literature. The healthy young adults in this study registered swallowing-pressure amplitudes in the range of only 10 to 15 percent of maximum isometric values, i.e., pressures of 50 to $100 \mathrm{mmHg}$ or 6 to $12 \mathrm{KPa}$, normalized with use of a standardized maximum isometric pressure value of $600 \mathrm{mmHg}$. Previous studies have observed a higher range of swallowing pressures approaching 50 percent of maximum values [9]. One possible explanation for this apparent discrepancy is that the current study did not involve radiation exposure, thereby allowing greater (and perhaps more representative) sampling of behavior across repeated task performances. However, this finding also suggests that the ideal magnitude of partial-pressure targets requires further study. Our study explored pressure profiles for half-maximum tongue-palate press tasks. It remains unknown whether tongue-palate press tasks targeting values in the 10 to 25 percent maximum range or those targeting values in the $>50$ percent maximum range may have slope profiles that differ from swallow pressureslope profiles to an even smaller extent. At this point, speculating on the ideal magnitude of partial-pressure task targets is clearly premature.

A second observation of importance in this study, as shown in Table 2, is that AHMAX tasks typically result in pressure patterns with little or no activity registered at the medial- and posterior-palate bulb locations. PHMAX tasks failed to involve anterior-bulb activation in a substantial number of our participants. This observation suggests that these tasks elicit pressure patterns that are questionably similar to those seen in normal swallowing tasks and further supports our concern that they may not have been sufficiently similar in slope characteristics to bolus swallows for inclusion in tongue resistance training protocols for swallow rehabilitation.

NESS yielded quite different pressure patterns than bolus swallows in this study. Specifically, both the rise and release phases of pressure were prolonged in the NESS task; consequently, the slopes of pressure rise and release at the anterior and posterior pressure bulbs, respectively, were found to be significantly less steep than those observed in bolus swallows. This finding suggests that there may be a particular salience to the parameter of pressure slope for swallowing function in the presence of a bolus and that it may not be adequate to focus exclusively on pressure range as a therapeutic target.

Inspection of the descriptive statistics in Table 2 shows that pressure slopes were steeper in the rise phase for DSS than for DSANEC, while the reverse phenomenon (steeper release) was observed with the nectar-thick stimuli. This difference, which was in the order of 20 to $120 \mathrm{mmHg} / \mathrm{s}$, requires further investigation because it may reflect an important modulatory factor in swallowing related to the precision of slope application and release across stimuli of differing viscosity. Furthermore, this difference suggests that pressure withdrawal by the posterior tongue may be a particularly important component in swallowing function. Pressure withdrawal has not, to our knowledge, been targeted in tongue resistance training protocols to date.

Clearly, a major issue with respect to the interpretation of this study is the fact that these pressure phenomena were measured in healthy adults. It remains unknown whether people with swallowing impairment involving tongue weakness will demonstrate similarities in pressure profiles across tasks in the same manner. A broader sampling of healthy adults across the age span will also be needed to establish normative ranges for adults of different ages with respect to pressure-slope parameters. These considerations precluded the direct application of these findings to patient populations. Nonetheless, we speculate that if patients with dysphagia are found to have deviant pressure slopes, a meaningful goal of swallowing intervention would be to attempt to establish more normative pressureslope patterns. The findings of the present study serve to inform such future efforts and suggest that an emphasis on tasks with slope characteristics similar to those seen in healthy swallowing may be an appropriate place to start. 


\section{CONCLUSIONS}

This study has identified a subset of tongue-pressure resistance training tasks, which display pressure-slope characteristics similar to those typically seen in water and nectar-thick liquid swallows. Saliva swallows were also found to yield pressure release-slope profiles that closely resemble those typically seen in water and nectar-thick liquid swallows. We conclude that protocols for tongue resistance training in individuals with dysphagia should incorporate these tasks to a substantial extent if a treatment goal is to include tasks similar to swallowing.

\section{ACKNOWLEDGMENTS}

\section{Author Contributions:}

Study concept and design: C. M. Steele.

Acquisition and processing of data: G. L. Bailey, S. M. Molfenter, E. M. Yeates.

Analysis and interpretation of data: C. M. Steele.

Critical revision of manuscript for important intellectual content:

S. M. Molfenter.

Statistical analysis: K. Grace-Martin.

Obtained funding: C. M. Steele.

Study supervision: C. M. Steele.

Financial Disclosures: A portion of these data, namely the pressure data on water and nectar-thick juice swallows, has been reported in a related article (Steele CM, Bailey GL, Molfenter SM. Tongue pressure modulation during swallowing: Water versus nectar-thick liquids. J Speech Lang Hear Res. 2010;53(2):273-83).

Dr. Steele discloses that KayPentax, the manufacturer of equipment discussed in this article, provided sponsorship for a series of workshops that she instructed. Otherwise, the authors have declared that no competing interests exist.

Funding/Support: This material was based on work supported by operating and career award grants from the Canadian Institutes of Health Research (grants 69521, 82668, 84534, and 83888). Additional funding support was provided by the Toronto Rehabilitation Institute and an Ontario Ministry of Research and Innovation Early Researcher Award to Dr. Steele. The authors acknowledge the support of the Toronto Rehabilitation Institute, which receives funding under the Provincial Rehabilitation Research Program from the Ministry of Health and Long-term Care in Ontario. The views expressed do not necessarily reflect those of the Ministry.

Additional Contributions: The authors gratefully acknowledge assistance provided by Melanie Moore, Rebecca Cliffe, and Nicole Buesselberg during data collection and processing. Erin M.Yeates is now with Saskatoon City Hospital, Saskatoon, Saskatchewan, Canada.

Institutional Review: The study was approved by the Toronto Rehabilitation Institute Research Ethics Board.

Participant Follow-Up: The authors do not plan to inform participants of the publication of this study. However, participants have been encouraged to check the hospital's research Web site for updated publications.

\section{REFERENCES}

1. Robbins J, Gangnon RE, Theis SM, Kays SA, Hewitt AL, Hind JA. The effects of lingual exercise on swallowing in older adults. J Am Geriatr Soc. 2005;53(9):1483-89.

[PMID: 16137276]

DOI:10.1111/j.1532-5415.2005.53467.x

2. Robbins J, Kays SA, Gangnon RE, Hind JA, Hewitt AL, Gentry LR, Taylor AJ. The effects of lingual exercise in stroke patients with dysphagia. Arch Phys Med Rehabil. 2007;88(2):150-58. [PMID: 17270511] DOI:10.1016/j.apmr.2006.11.002

3. El Sharkawi A, Ramig L, Logemann JA, Pauloski BR, Rademaker AW, Smith CH, Pawlas A, Baum S, Werner C. Swallowing and voice effects of Lee Silverman Voice Treatment (LSVT): A pilot study. J Neurol Neurosurg Psychiatry. 2002;72(1):31-36. [PMID: 11784821] DOI:10.1136/jnnp.72.1.31

4. Burkhead LM, Sapienza CM, Rosenbek JC. Strength-training exercise in dysphagia rehabilitation: Principles, procedures, and directions for future research. Dysphagia. 2007;22(3): 251-65. [PMID: 17457549] DOI:10.1007/s00455-006-9074-z

5. Lazarus C, Logemann JA, Huang CF, Rademaker AW. Effects of two types of tongue strengthening exercises in young normals. Folia Phoniatr Logop. 2003;55(4):199-205. [PMID: 12802092]

DOI:10.1159/000071019

6. Wheeler KM, Chiara T, Sapienza CM. Surface electromyographic activity of the submental muscles during swallow and expiratory pressure threshold training tasks. Dysphagia. 2007;22(2):108-16. [PMID: 17294298]

DOI:10.1007/s00455-006-9061-4

7. Shaker R, Easterling C, Kern M, Nitschke T, Massey B, Daniels S, Grande B, Kazandjian M, Dikeman K. Rehabilitation of swallowing by exercise in tube-fed patients with pharyngeal dysphagia secondary to abnormal UES opening. Gastroenterology. 2002;122(5):1314-21. [PMID: 11984518$]$ DOI:10.1053/gast.2002.32999

8. Clark HM. Neuromuscular treatments for speech and swallowing: A tutorial. Am J Speech Lang Pathol. 2003;12(4): 400-15. [PMID: 14658992] DOI:10.1044/1058-0360(2003/086)

9. Nicosia MA, Hind JA, Roecker EB, Carnes M, Doyle J, Dengel GA, Robbins J. Age effects on the temporal evolution of isometric and swallowing pressure. J Gerontol A Biol Sci Med Sci. 2000;55(11):M634-40. [PMID: 11078092] 
10. Winstein CJ, Rose DK, Tan SM, Lewthwaite R, Chui HC, Azen SP. A randomized controlled comparison of upperextremity rehabilitation strategies in acute stroke: A pilot study of immediate and long-term outcomes. Arch Phys Med Rehabil. 2004;85(4):620-28. [PMID: 15083439] DOI:10.1016/j.apmr.2003.06.027

11. Monfils MH, Plautz EJ, Kleim JA. In search of the motor engram: Motor map plasticity as a mechanism for encoding motor experience. Neuroscientist. 2005;11(5):471-83.

[PMID: 16151047] DOI:10.1177/1073858405278015

12. Yeates EM, Molfenter SM, Steele CM. Improvements in tongue strength and pressure-generation precision following a tongue-pressure training protocol in older individuals with dysphagia: Three case reports. Clin Interv Aging. 2008; 3(4):735-47. [PMID: 19281066]

13. Steele CM, Bailey GL, Molfenter SM. Tongue pressure modulation during swallowing: Water versus nectar-thick liquids. J Speech Lang Hear Res. 2010;53(2):273-83. [PMID: 20008678] DOI:10.1044/1092-4388(2009/09-0076)

14. Steele CM, Huckabee ML. The influence of orolingual pressure on the timing of pharyngeal pressure events. Dysphagia. 2007;22(1):30-36. [PMID: 17024546$]$ DOI:10.1007/s00455-006-9037-4

15. Huckabee ML, Steele CM. An analysis of lingual contribution to submental surface electromyographic measures and pharyngeal biomechanics during effortful swallow. Arch Phys Med Rehabil. 2006;87(8):1067-72.
[PMID: 16876551]

DOI:10.1016/j.apmr.2006.04.019

16. Hiiemae KM, Palmer JB. Tongue movements in feeding and speech. Crit Rev Oral Biol Med. 2003;14(6):413-29.

[PMID: 14656897] DOI:10.1177/154411130301400604

17. White R, Cotton SM, Hind J, Robbins J, Perry A. A comparison of the reliability and stability of oro-lingual swallowing pressures in patients with head and neck cancer and healthy adults. Dysphagia. 2009;24(2):137-44.

[PMID: 18956230]

DOI:10.1007/s00455-008-9181-0

18. Bennett JW, Van Lieshout PH, Pelletier CA, Steele CM. Sipsizing behaviors in natural drinking conditions compared to instructed experimental conditions. Dysphagia. 2009;24(2): 152-58. [PMID: 18841414$]$ DOI:10.1007/s00455-008-9183-y

Submitted for publication May 28, 2009. Accepted in revised form May 4, 2010.

This article and any supplementary material should be cited as follows:

Steele CM, Bailey GL, Molfenter SM, Yeates EM, GraceMartin K. Pressure profile similarities between tongue resistance training tasks and liquid swallows. J Rehabil Res Dev. 2010;47(7):651-60. DOI:10.1682/JRRD.2009.05.0068 